\title{
Luminal Endocytosis and Intracellular Targeting by Acinar Cells during Early Biliary Pancreatitis in the Opossum
}

\author{
Markus M. Lerch, Ashok K. Saluja, Michael Rünzi, Rajinder Dawra, and Michael L. Steer \\ The Pancreas Group, Department of Surgery and Harvard Digestive Diseases Center, Beth Israel Hospital \\ and Harvard Medical School, Boston, Massachusetts 02215
}

\begin{abstract}
Cell necrosis in acute experimental pancreatitis is preceded by a redistribution of digestive enzymes into a lysosomal subcellular compartment. We have investigated whether endocytosis from the acinar cell lumen might contribute to this disturbance of intracellular compartmentation. In an animal model of pancreatitis involving pancreatic bile duct ligation in opossums, we have studied in vivo endocytosis of dextran 40 and $\left[{ }^{14} \mathrm{C}\right] d$ extran 70 , cationized ferritin, and horseradish peroxidase from the apical surface of acinar cells before the onset of necrosis. Marker solutions were instilled into the pancreatic duct of anesthetized animals at physiological pressure. Tissue samples obtained at intervals of up to $60 \mathrm{~min}$ after instillation of markers were studied by electron microscopy and electron microscope autoradiography. All markers were taken up by acinar cells in control animals and in animals with obstructed pancreatic bile ducts. Markers for membrane-mediated endocytosis (cationated ferritin and horseradish peroxidase) were transported to lysosomes in both groups. In contrast, the fluidphase tracer dextran was transported to the secretory pathway in controls but to lysosomes after duct ligation. Since dextran and luminally present secretory proteins can be expected to follow the same route after endocytosis, our findings suggest that altered intracellular targeting of endocytosed proteases might be one mechanism by which digestive zymogens reach an intracellular compartment in which premature activation can occur. This phenomenon may be a critical and early event in the pathogenesis of biliary pancreatitis. (J. Clin. Invest. 1995. 95:2222-2231.) Key words: cationized ferritin - dextran • endocytosis • horseradish peroxidase $\cdot$ lysosome $\cdot$ intracellular targeting
\end{abstract}

\section{Introduction}

Passage of a calculus through the biliary tract is generally believed to be the event that triggers acute gallstone-induced pan-

Address correspondence to Michael L. Steer, M.D., Department of Surgery, Beth Israel Hospital, 330 Brookline Avenue, Boston, MA 02215. Phone: 617-735-4261; FAX: 617-735-2978. M. M. Lerch's present address is Abteilung Molekularbiologie, Max-Plank-Institut für Biochemie, Am Klopferspitz 18A, 82152 Martinsried, Germany.

Received for publication 25 March 1994 and in revised form 5 December 1994

J. Clin. Invest.

(C) The American Society for Clinical Investigation, Inc.

0021-9738/95/05/2222/10 \$2.00

Volume 95, May 1995, 2222-2231 creatitis (1). Although it was previously believed that bile reflux into the pancreatic duct must occur in order for pancreatitis to develop (2), recent experimental (3) and clinical $(4,5)$ evidence suggests that pancreatic outflow obstruction, rather than reflux of bile, is the actual event that triggers acute pancreatitis. Experimental studies also suggest that the development of pancreatic necrosis during pancreatitis is preceded by the premature and intraglandular activation of pancreatic digestive enzyme zymogens (6). The mechanisms, however, by which pancreatic duct outflow obstruction leads to zymogen activation is not known.

The earliest cell biological and morphological changes observed during the evolution of pancreatitis appear to occur in acinar cells. Under physiologic conditions, acinar cells actively internalize elements of the apical (i.e., luminal) plasmalemma as well as fluid-phase content from the ductal space (7), and during acute pancreatitis, activated pancreatic digestive enzymes have been detected within the pancreatic ducts $(8-10)$. These observations suggested to us that alterations in luminal endocytosis and/or intracellular transport of internalized material may play an important role in the evolution of acute pancreatitis. In the current communication, we report the results of experiments that evaluated this issue using a model of acute necrotizing pancreatitis that is induced by obstructing the pancreatic bile duct (P-BD) ${ }^{1}$ of the opossum. Markers for affinitymediated, receptor-mediated, and fluid-phase endocytosis, which can be visualized by electron microscopy, were instilled into the duct at physiologic pressure in control animals and in animals after P-BD ligation for up to $3 \mathrm{~h}$ (the early phase of pancreatitis that precedes acinar cell necrosis).

We report that luminal endocytosis continues for at least as $3 \mathrm{~h}$ after P-BD obstruction, but that intracellular transport of internalized fluid-phase luminal content is altered. Rather than being targeted to secretory elements of the cell, as occurs under physiologic conditions, fluid-phase material is transported to the lysosomal compartment after duct obstruction. This continuous uptake and subsequent rerouting of internalized material may play an important role in the evolution of acute pancreatitis.

\section{Methods}

Animal preparation. Randomly trapped opossums (Didelphis virginia) of either sex weighing $2.5-4.2 \mathrm{~kg}$ were obtained from R-Zoo (Neshkoro, WI). They were individually housed in steel, temperature-controlled $\left(23 \pm 3^{\circ} \mathrm{C}\right)$ cages, exposed to a 12-h-light/12-h-dark cycle, and fed a standard laboratory chow containing $21 \%$ protein, $7 \%$ fat, and $6 \%$ fiber (Purina Mills, St. Louis, MO), with water ad libitum. The follow-

1. Abbreviations used in this paper: $\mathrm{BD}$, bile duct; cat-ferritin, cationized ferritin; HRP, horseradish peroxidase; P-BD, pancreatic BD. 


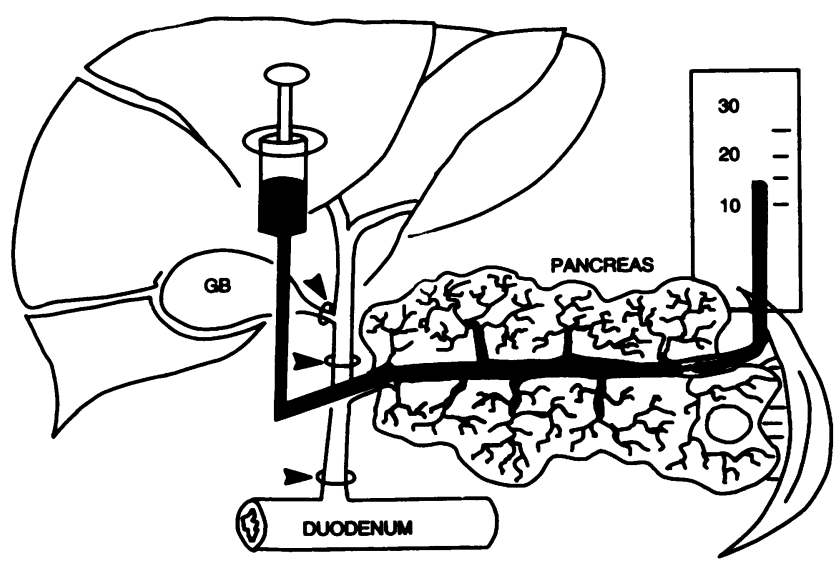

Figure 1. Experimental procedure. After the surgical creation of a ligated segment of the P-BD (arrowheads), a tube was inserted into the pancreatic duct. A second tube inserted into the duct at the tail of the pancreas drained the tracer solution after the entire ductal system had been filled and allowed maintenance of the perfusion pressure within physiologic bounds. In control animals only the cystic duct was ligated (upper arrowhead) to prevent the gallbladder $(G B)$ form serving as a bile reservoir, and the common duct was ligated above the insertion of the pancreatic duct, leaving pancreatic secretion unimpaired (middle arrowhead).

ing experimental protocol was performed after approval by the animal care committee of Beth Israel Hospital.

After an overnight fast, the animals were anesthetized with an intraperitoneal injection of pentobarbital $(50 \mathrm{mg} / \mathrm{kg})$, and anesthesia was maintained throughout the experiment by periodic intravenous bolus injections of pentobarbital ( $3-5 \mathrm{mg}$ ). Celiotomy was performed through a midline incision, and the cystic duct was ligated in all animals to prevent the gallbladder from serving as a bile reservoir. The animals were then randomly divided into three groups. The control group ( $n$ $=17$ ) underwent ligation of the bile duct (BD) proximal to its junction with the pancreatic duct. As a result, these control animals experienced only BD obstruction, and pancreatic secretion continued to drain freely into the duodenum. The two experimental groups underwent ligation of the common P-BD at its point of entry into the duodenum, for either 1 $\mathrm{h}(n=21)$ or $3 \mathrm{~h}(n=27)$. As a result, these animals experienced combined pancreatic and bile duct obstruction. P-BD obstruction in the opossum is known to cause acute necrotizing pancreatitis, but acinar cell necrosis is not observed in this model until the duct has been obstructed for at least $6 \mathrm{~h}$ (11). Thus, after only 1 or $3 \mathrm{~h}$ of duct obstruction, some acinar cells show alterations that affect the endoplasmic reticulum, the cellular polarity, and the apical morphology, but cell necrosis has not yet occurred.

After BD ligation in the control group or P-BD ligation for 1 or 3 $h$ in the experimental groups, the P-BD was ligated at the duodenum in the control group and the BD was ligated proximal to its junction with the pancreatic duct in the experimental groups. This created a blind segment of common P-BD through which a catheter (PE-50; Clay Adams, Parsippany, NJ) was inserted into the pancreatic duct (Fig. 1). Then bile and pancreatic juice were allowed to drain from this catheter in order to empty the ductal system as completely as possible. To vent the ductal space during the instillation of tracer solution, the pancreatic duct in the tail of the pancreas was cannulated with polyethylene tubing (PE-50) that was secured with a single suture of $3 / 0$ silk. The end of this vent tubing was elevated to $15 \mathrm{~cm}$ above the papilla of Vater.

After duct obstruction and cannulation as previously described, the duct was instilled with marker solutions in a retrograde fashion, gently filling the duct via the cannula in the common P-BD over a period of $3 \mathrm{~min}$. Throughout this time, intraductal pressure was maintained within a physiologic range $\left(15-23 \mathrm{cmH}_{2} \mathrm{O}\right)$ since the vent cannula pre ented intraductal pressure from exceeding $15 \mathrm{cmH}_{2} \mathrm{O}$. The filling vime, which corresponded to the duct capacity under these conditions, ranged from $1.8 \pm 0.3 \mathrm{ml}$ in control animals to $2.7 \pm 0.4 \mathrm{ml}$ in animals a er $\mathrm{P}$ BD obstruction. After the duct had been filled with marker solutions for $3 \mathrm{~min}$, infusion was halted and ductal fluid was allowed to drain freely to the outside via the cannula in the P-BD. At selected times $(0-$ $60 \mathrm{~min}$ ), tissue samples were collected from the head, body, and tail of the pancreas, immediately immersed in iced fixative, and processed for electron microscopy.

Because luminal endocytosis was the object of our study, we took the following three precautions to avoid the possibility that basolateral endocytosis might occur: $(a)$ to prevent disruption of luminal tight junctions, we kept the instillation pressure within the physiologic range and inserted the tail duct venting catheter previously mentioned; $(b)$ a dye was added to the tracer solutions, and when stereo microscopic examination before tissue harvesting indicated that the solution had escaped into the interstitial space, samples were excluded from further consideration; and $(c)$ when, on electron microscopic examination, either interstitial junctions were found disrupted or tracer was detected in the interstitial space, the specimen was excluded form further evaluation.

Marker solutions. Three markers for endocytosis, all easily visualized by electronmicroscopy, were used. Dextran 40 was used to study fluid-phase endocytosis since it does not bind, either specifically or nonspecifically, to cellular membranes (12). Membrane-mediated endocytosis was investigated using cationized ferritin (cat-ferritin) and horseradish peroxidase (HRP). The former binds to sialic acid and other negatively charged residues on cell membranes (13) and thus serves as a marker for so-called affinity-mediated endocytosis of surface membrane. HRP binds to mannose/ $N$-acetylglucosamine receptors on the cell surface $(14,15)$ and thus serves as a marker for receptor-mediated endocytosis. HRP can also be internalized with bulk fluid solutes.

All marker solutions were prepared using $150 \mathrm{mM}$ Tris- $\mathrm{HCl}$ buffered (pH 8.3) to prevent precipitation with ductal content. Cat-ferritin (Miles-Yeda, Rehovot, Israel) and HRP (Sigma Chemical Co., St. Louis, MO) were used at concentrations of $10 \mathrm{mg} / \mathrm{ml}$. Dextran 40 (35,600 or $38,800 \mathrm{MW}$; Sigma Chemical Co. or Pharmacid Diagnostics AB, Uppsala, Sweden) was used at a concentration of $200 \mathrm{mg} / \mathrm{ml}$. All solutions were labeled with blue dextran $(10 \mathrm{mg} / \mathrm{ml})$ to allow for stereo microscopic evaluation of the distribution within the ductal system during filling. Tissue areas with possible interstitial infiltration of the marker (16) were not selected for subsequent morphologic examination, as previously indicated. As an additional fluid-phase control for the uptake and intercellular processing of dextran 40 , we performed a series of experiments with $\left[{ }^{14} \mathrm{C}\right.$ ] dextran (70,000 MW; Sigma Chemical Co.; 250 $\mu \mathrm{Ci} / \mathrm{ml}$ tracer solution). These studies allowed for an evaluation of the intracellular distribution of the fluid-phase marker not only by morphologic criteria, but also by localizing and quantitating silver grains in EM autoradiograms as described in the following section. For these experiments, BD controls and animals after P-BD obstruction for $3 \mathrm{~h}$ were used.

Microscopy. Two different fixation procedures were used for electron microscopy. First, strips of pancreas measuring $1.0 \times 0.5 \mathrm{~mm}$ were immediately fixed in $125 \mathrm{mM}$ phosphate buffer ( $\mathrm{pH} \mathrm{7.4)}$ containing $2 \%$ glutaraldehyde and $4 \%$ formaldehyde for $90 \mathrm{~min}$, rinsed extensively in the same buffer, and postfixed in $2 \% \mathrm{OsO}_{4}$ either with or without addition of $1.5 \%$ potassium ferrocyanide. Alternatively, an en bloc method previously reported to be especially suited for the visualization of dextrans (12) was used. In brief, three stock solutions (A: $3 \%$ formaldehyde plus $5 \%$ glutaraldehyde; $\mathrm{B}: 2 \% \mathrm{OsO}_{4}$; $\mathrm{C}$ : saturated lead citrate solution) were mixed at $4^{\circ} \mathrm{C}$ immediately before use at a ratio of $3: 2: 1$ volumes, and tissue strips were immersed for $2 \mathrm{~h}$ before washing with phosphate buffer. Tissue blocs were dehydrated in ethanol and embedded in Epon 812. Semithin sections were stained with methylene blue and examined with a light microscope. Selected areas, chosen for detailed study, were thin sectioned using an ultratome (LKB Wallac, Turku, Finland), picked up on uncoated copper grids, double stained 
with uranyl acetate and lead citrate, and examined on a Philips 300 transmission electron microscope (Philips Eletronic Instruments, Mahwah, NJ). Specimens fixed by the en bloc method were viewed either unstained or stained with uranyl acetate alone.

Cytochemistry. HRP was visualized by cytochemistry with 3,3'diaminobenzidine $(1 \mathrm{mg} / \mathrm{ml})$ as described by Graham and Karnovsky (17) with the addition of $0.01 \% \mathrm{H}_{2} \mathrm{O}_{2}$ in the second incubation. Acid phosphatase as a marker enzyme for secondary lysosomes was visualized according to the cytidine $2^{\prime} 3^{\prime}$-monophosphate technique (18).

Electron microscopic autoradiography. In experiments with $\left[{ }^{14} \mathrm{C}\right]-$ dextran, plastic-embedded tissue specimens were cut into thin sections and coated with Ilford L4 emulsion (Polysciences, Warrington, PA) using a platinum wire loop technique. After exposure for $100 \mathrm{~d}$ in the dark, they were developed in Microdol X (Eastman Kodak Co., Rochester, NY) and contrasted with standard techniques (19).

Quantitation of endocytosis. Cross sections of pancreatic acinar cells that included a segment of luminal plasmalemma no shorter than $2 \mu \mathrm{m}$ in addition to a segment of nucleus no smaller than $3 \mu \mathrm{m}$ in diameter were randomly chosen, photographed, and printed on photographic paper at a calibrated magnification of 16,000 covering the entire section of the cell. Prints of each cell were coded. The number of organelles with positive evidence of the respective marker was determined in a blinded fashion by two independent investigators and expressed per $100 \mu \mathrm{m}^{2}$ of cell surface (graphic tablet, model 4933; Tektronix Inc., Beaverton, OR; IBM-PC). Three types of organelles were classified for evaluation using morphologic criteria: Endocytic vesicles were defined as coated or uncoated vesicles that had a diameter of $\leq 1 \mu \mathrm{m}$ and were in the vicinity ( $\leq 2.5 \mu \mathrm{m}$ ) of the apical plasmalemma. Secretory vesicles included zymogen granules with a completely circular shape, a diameter between 0.5 and $1.5 \mu \mathrm{m}$, and under the conditions of our fixation procedures, a dark and completely homogeneous content. The secretory vesicle group also included condensing secretory vacuoles that, because they are more variable in their circular shape and density of content, had to be localized within $3 \mu \mathrm{m}$ of the trans face of a Golgi apparatus to qualify for morphometric evaluation. Lysosomal vesicles were defined as acid phosphatase-positive structures; this group included secondary lysosomes and autophagic vacuoles of variable size (being generally larger in duct-obstructed animals ). According to these definitions, 93\% of vesicles could be classified into the appropriate category. The remainder were excluded form further evaluation. A minimum of 100 cells per experiment were evaluated in this manner. The variation between the two investigators who independently evaluated the samples was $<3 \%$. To evaluate electron microscope autoradiograms, the distribution of silver grains over the respective intracellular areas was determined according to standard techniques (20). For these studies, a silver grain was defined as being localized over a vesicle or organelle if the distance between the center of the grain and the outer limit of the respective compartment was $\leq 0.2 \mu \mathrm{m}$ (21). Label over the nuclear region was regarded as background and was subtracted form vesicular counts.

Data at the different time intervals after perfusion represent means \pm SEM from at least three separate experiments. The absence of error bars indicates that differences were too small to illustrate. The experimental groups were compared with the control group by analysis of variance, and differences were considered significant if the $q$ value was more than the critical value when $\alpha \leq 0.05$. Data that were found to differ significantly from the control group are indicated by asterisks in the figures.

All chemicals were of the highest purity available and were obtained from Sigma Chemical Co.), Polysciences Ted Pella (Redding, CA) and EMS (Fort Washington, PA) unless otherwise indicated in the text.

\section{Results}

Endocytosis of cat-ferritin. Cat-ferritin binds to negatively charged membrane domains and is therefore regarded as a marker for affinity-mediated endocytosis. At the earliest time interval evaluated after instillation into the pancreatic duct, the tracer was found lining the apical plasmalemma of pancreatic acinar cells (Fig. $2 A$ ). By 5 min, coated and uncoated pits containing cat-ferritin were found to separate from the apical membrane and the tracer could be demonstrated in small endocytic vesicles (Fig. $2 B$ ). After transport away from the lumen in endocytic vesicles (Fig. $2 C$ ), cat-ferritin was localized with increasing frequency in small lysosomal vesicles located predominantly in the supranuclear region of the cell (Fig. $2 \mathrm{D}$ ). The secretory pathway was invariably spared, and neither postGolgi condensing vacuoles nor secretory granules contained cat-ferritin. P-BD obstruction for 1 or $3 \mathrm{~h}$ did not alter the target organelle for the tracer. Lysosomes remained the primary organelle for cat-ferritin localization after P-BD ligation (Fig. $2 F$ ). In addition, and particularly after $3 \mathrm{~h}$ of duct obstruction, cat-ferritin was noted to be present in autophagic vacuoles (Fig. $2 D$ ).

Endocytosis of HRP. HRP binds to mannose/ $N$-acetylglucosamine receptors on the cell surface $(14,15)$ and is therefore regarded as a marker for partially receptor-mediated endocytosis. The normal acinar lumen is shown in Fig. $3 \mathrm{~A}$. Instillation of HRP into the duct caused a distension of the acinar lumen, which was filled with dark HRP reaction product. The integrity of microvilli and intercellular junctions was not altered (Fig. 3 $B)$. Endocytosis from the lumen occurred in small endocytic vesicles. Lysosomes in control as well as experimental animals could easily be identified on the basis of their acid phosphatase content (Fig. $3 \mathrm{D}$ ). HRP was found in these structures after endocytosis regardless of whether or not the P-BD was ligated before the instillation of the tracer into the duct (Fig. $3 E$ ). After $3 \mathrm{~h}$ of duct obstruction, large cytoplasmic vacuoles represented an additional target organelle for HRP reaction product (Fig. $3 F$ ).

Endocytosis of dextran. Dextran 40 undergoes endocytosis with bulk fluid solutes without specific or nonspecific binding to cellular membranes. It is therefore regarded as a fluid-phase marker for luminal content. The physiological content in the acinar lumen is shown in Fig. $4 A$. Instillation of dextran 40 into the duct resulted in a dense granular reaction product that appeared to mix with the normal content (Fig. $4 \mathrm{~B}$ ). The tight junctions between adjacent acinar cells were not affected by the perfusion procedure. After its endocytosis from the lumen, dextran 40 was localized predominantly in vesicles of the secretory pathway, i.e., condensing vacuoles and zymogen granules (Fig. $4 C$ ). P-BD ligation altered this transport and targeting significantly. As early as $1 \mathrm{~h}$ after duct ligation, dextran 40 was found almost exclusively in lysosomes (Fig. 4 D). Large cytoplasmic vacuoles could be readily identified in acinar cells of duct-obstructed animals whose pancreatic duct was not filled with marker solution (Fig. $4 E$ ). In duct-obstructed animals whose duct was filled with marker solution, these vacuoles became the principal target organelle for dextran 40 (Fig. $4 F$ ).

When $\left[{ }^{14} \mathrm{C}\right]$ dextran was used, the presence of silver grains greatly facilitated the localization. The overlying photoemulsion, however, made the classification of $\sim 15 \%$ of vesicles arbitrary, and these structures had to be excluded from morphometry. Most striking was the significant presence of silver grains over zymogen granules in control animals (Fig. 5) and their nearly complete absence over the secretory compartment after $3 \mathrm{~h}$ of P-BD obstruction. 

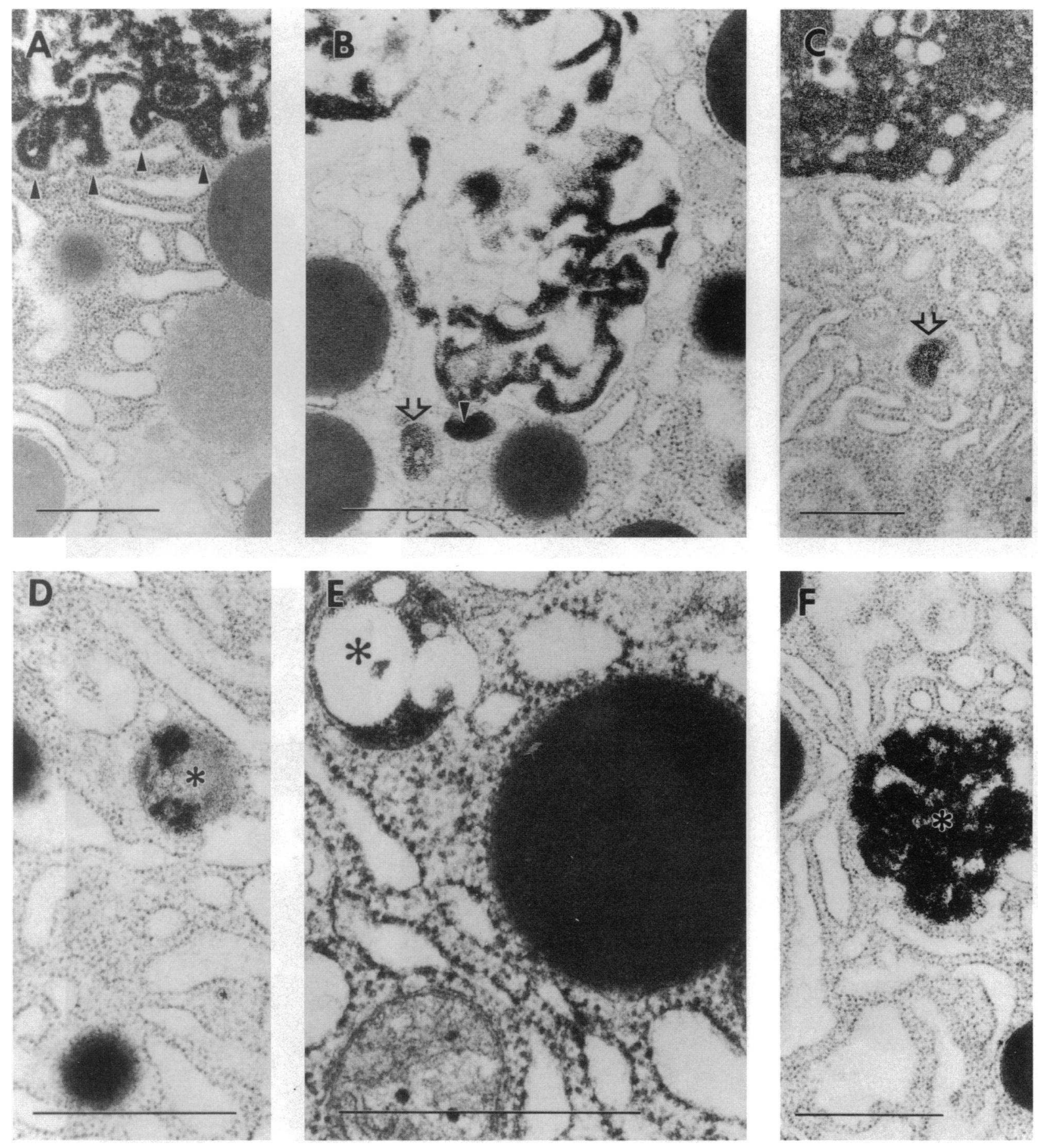

Figure 2. Endocytosis of cat-ferritin. Cat-ferritin was instilled into the pancreatic duct at physiologic pressure as described in the text. Micrographs from tissue specimens at different time intervals after instillation represent findings from three or more animals. Calibration bars represent $1 \mu \mathrm{m}$. ( $A$ ) The acinar lumen and its protrusions are completely lined with cat-ferritin (arrowheads). No endocytosis of the tracer is yet observed (control, 1 min after instillation). (B) The luminal content of cat-ferritin appears more inhomogeneous and is increasingly concentrated along the luminal plasmalemma. Small vesicles can be observed either budding from the membrane (arrowhead) or already in the apical portion of the cell (open arrow) (control, 5 min after instillation). ( $C$ ) Endocytic vesicles containing cat-ferritin (open arrow) move progressively toward the center of the cell (control, $10 \mathrm{~min}$ after instillation). (D) In control animals the principal target organelles after endocytosis from the lumen are small lysosomes (asterisk) (control, $30 \mathrm{~min}$ after instillation). ( $E$ ) Duct ligation alters neither the endocytosis of cat-ferritin nor its intracellular targeting to lysosomes (asterisk). The adjacent zymogen granule is part of the secretory pathway and does not contain any tracer (1-h duct ligation, 30 min after instillation). $(F)$ With longer time intervals after duct ligation, the developing cytoplasmic vacuoles (asterisk) become a more frequent and conspicuous target for cat-ferritin after endocytosis (3-h duct ligation, $45 \mathrm{~min}$ after instillation). 



Figure 3. Endocytosis of HRP. HRP was instilled into the pancreatic duct at physiologic pressure as described in the text. Micrographs from tissue specimens at different time intervals after perfusion represent findings from three or more animals. Calibration bars represent $1 \mu \mathrm{m}$. ( $A$ ) Without tracer instillation, the acinar lumen appears empty, with microvilli protruding from the apical membrane (control, no instillation). ( $B$ ) HRP fills the entire lumen with dark reaction product but does not affect tight junctions (arrowheads) between acinar cells (control, 1 min after instillation). (C) Occasionally, HRP mixes with the secretory content of a zymogen granule (arrow) that is in the process of exocytosis (control, 1 min after instillation). ( $D$ ) In control animals lysosomes (asterisk) are not always conspicuous but can be identified by acid phosphatase cytochemistry (control, no instillation). (E) In controls and animals after duct ligation HRP reaction product is localized in lysosomes (asterisk) after endocytosis (1-h duct ligation, $30 \mathrm{~min}$ after instillation). $(F)$ With increasing duration of duct ligation, cytoplasmic vacuoles (top half of panel) form, increase in size, and become a target organelle for HRP (3-h duct ligation, 60 min after instillation). 

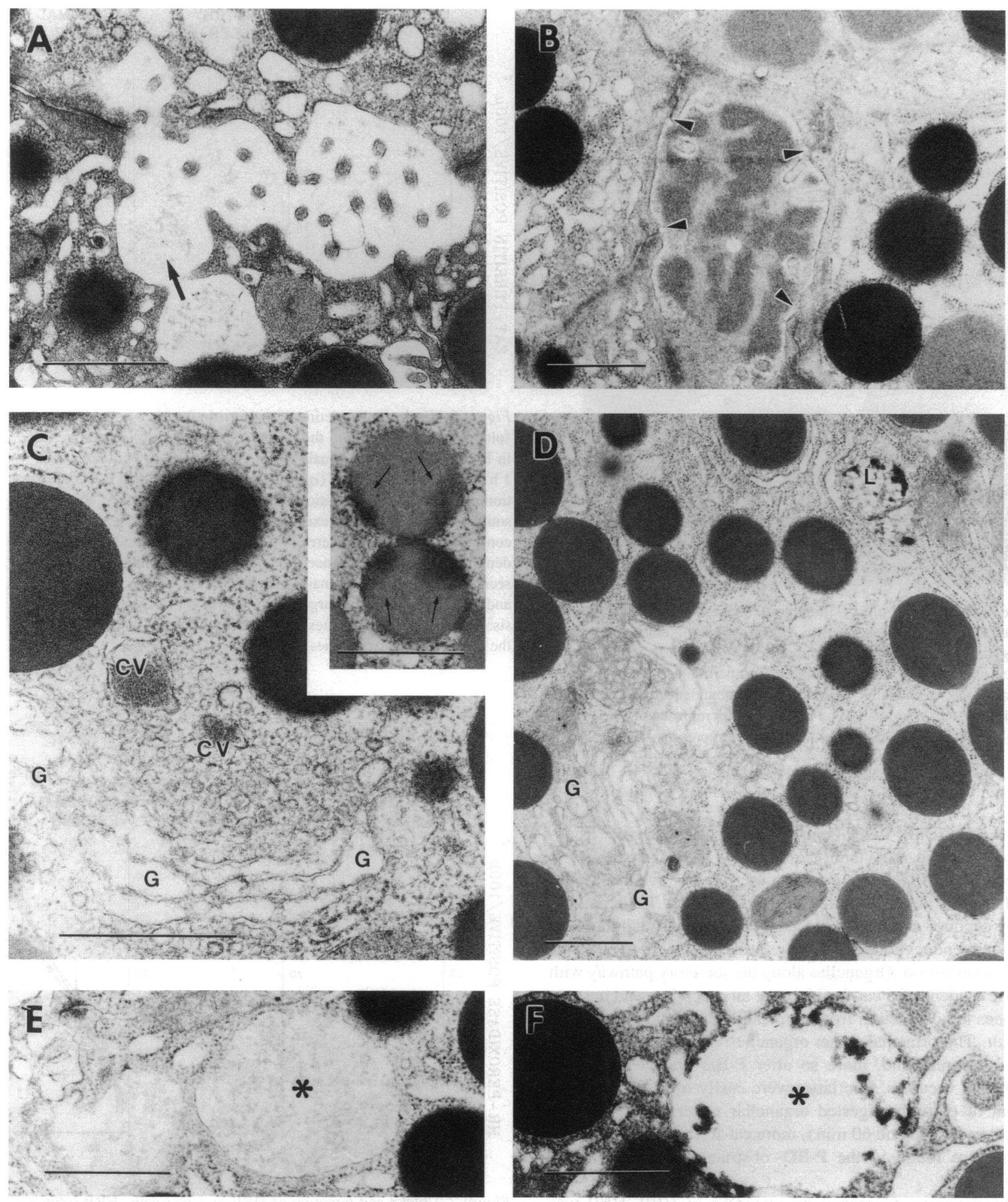

Figure 4. Endocytosis of dextran 40. Dextran 40 was instilled into the pancreatic duct at physiologic pressure as described in the text. Micrographs from tissue specimens at different time intervals after perfusion represent findings from three or more animals. Calibration bars represent $1 \mu \mathrm{m}$. $(A)$ The acinar lumen of an unstimulated control animal without instillation of the tracer is shown to illustrate its physiologic appearance with secretory content and intact microvilli. Occasionally, the fusion/fission of a zymogen granule (arrow) can be observed (control, no tracer instillation). $(B)$ The instillation of dextran 40 fills and distends the lumen with dark granular material but leaves microvilli and intercellular junctions (arrowheads) intact (control, $5 \mathrm{~min}$ after instillation). (C) Already within the first $10 \mathrm{~min}$ after perfusion, dextran 40 can be localized in the secretory pathway of control animals. Here it is found in condensing vacuoles $(C V)$ leaving the Golgi region $(G)$ and in zymogen granules (inset, arrows) (control, 15 min after instillation). (D) P-BD ligation alters the intracellular targeting of endocytic dextran rapidly. The tracer is completely absent from the Golgi region $(G)$ and secretory vesicles and is found instead in lysosomes $(L)$ (1-h duct ligation, 15 min after instillation). (E) Large cytoplasmis vacuoles (asterisk) develop in pancreatic acinar cells regardless of whether or not a tracer solution was perfused through the duct (control, no instillation). $(F)$ These cytoplasmic vacuoles (asterisk) become a principal target organelle for dextran 40 (3-h duct ligation, 30 min after instillation). 




Figure 5. Endocytosis of $\left[{ }^{14} \mathrm{C}\right]$ dextran. $\left[{ }^{14} \mathrm{C}\right]$ dextran was instilled into the pancreatic duct as described in Methods, and the animal (control, BD ligation) was sacrificed $60 \mathrm{~min}$ later. As opposed to P-BD-obstructed animals in which the secretory compartment was devoid of $\left[{ }^{14} \mathrm{C}\right]$ dextran, silver grains were detected in abundance over zymogen granules in control animals. The micrograph is representative of three animals in this group, and the calibration bar represents $1 \mu \mathrm{m}$.

Quantitation of uptake and targeting of cat-ferritin. Endocytic vesicles in the vicinity of the cell apex were found to contain cat-ferritin at the earliest time interval evaluated after instillation of the tracer into the duct (Fig. 6). Their number slowly declined over the subsequent $60 \mathrm{~min}$, but small numbers of labeled endocytic vesicles were still seen at the end of the observation period. Organelles along the secretory pathway with positive evidence for cat-ferritin were an exception, and in some instances the distinction from atypically shaped lysosomes was difficult. The principal target organelles containing cat-ferritin were lysosomes and, more so after P-BD obstruction, small autophagic vacuoles. The latter were easily identified by their content of partially digested organellar material. At the later time intervals ( 45 and $60 \mathrm{~min}$ ), more cat-ferritin-positive lysosomes were found in the P-BD-obstructed animals than in controls.

Quantitation of uptake and targeting of HRP. HRP was internalized and transported in a manner similar to that observed with cat-ferritin. After endocytosis, the lysosomal compartment was the principal target organelle, regardless of whether or not the P-BD had previously been obstructed (Fig. 7). Differences in the number of positive lysosomes between obstructed and nonobstructed animals could not be demonstrated. This may be due to the greater variation among animals and the lower total number of HRP-labeled lysosomal vesicles per surface area as

\section{SECRETORY VESICLES}
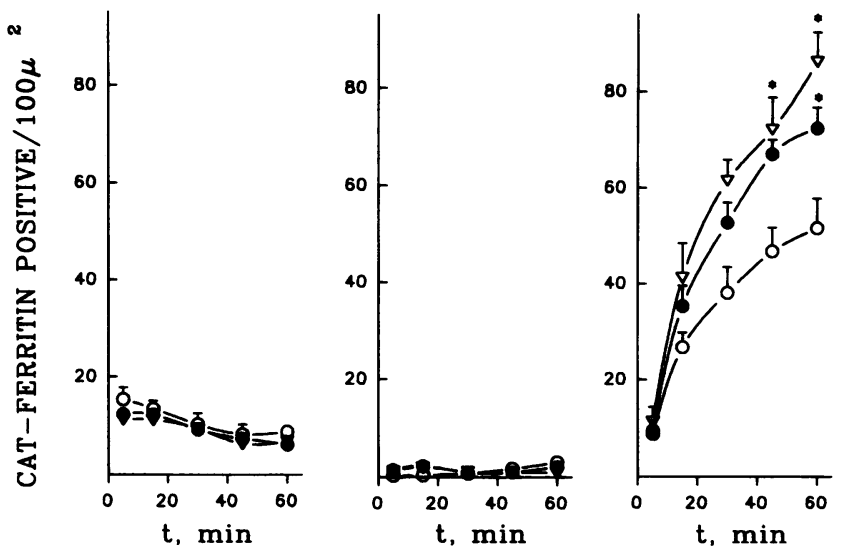

Figure 6. Uptake and targeting of cat-ferritin in acinar cells. Cat-ferritin solution was instilled into the pancreatic duct at physiologic pressure in BD-ligated control animals (open circles) and in opossums after $1 \mathrm{~h}$ (closed circles) or $3 \mathrm{~h}$ (open triangles) of combined P-BD obstruction. Morphometric evaluation of the endocytic, secretory, and lysosomal compartments was executed as described in the text. Whereas continuous uptake of cat-ferritin in the P-BD-obstructed groups can be demonstrated by its presence in endocytic vesicles, the amount in the secretory compartment remains negligible in all groups. Lysosomes are and remain the principal target organelle for cat-ferritin after endocytosis. Asterisks indicate values significantly different $(\alpha \leq 0.05)$ from the respective control values.

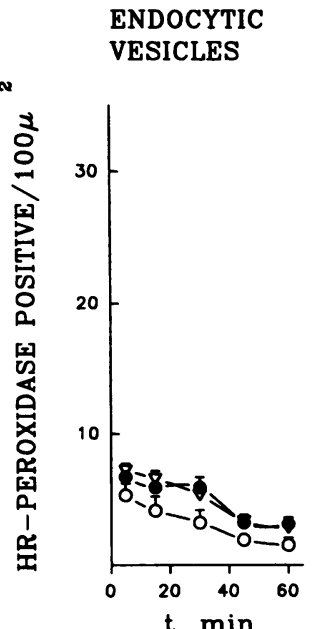

\section{SECRETORY VESICLES}
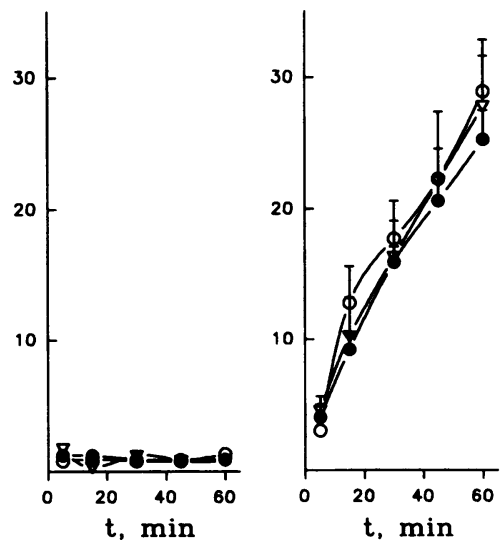

Figure 7. Uptake and targeting of HRP in acinar cells. HRP solution was instilled into the pancreatic duct at physiologic pressure in BDligated control animals (open circles) and in opossums after $1 \mathrm{~h}$ (closed circles) or $3 \mathrm{~h}$ (open triangles) of combined P-BD obstruction. Morphometric evaluation of the endocytic, secretory, and lysosomal compartments was executed as described in the text. In a manner comparable to cat-ferritin, the uptake of HRP in the P-BD-obstructed groups continued. The amount of reaction product in the secretory compartment was negligible in all groups at all intervals, and structures of lysosomal origin were the principal target organelle for HRP after endocytosis. 


\section{ENDOCYTIC VESICLES}

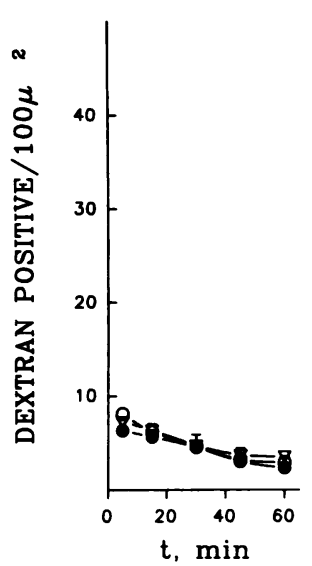

SECRETORY VESICLES

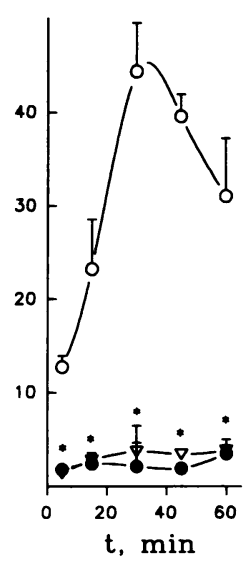

LYSOSOMAL VESICLES



Figure 8. Uptake and targeting of dextran 40 in acinar cells. Dextran 40 solution was instilled into the pancreatic duct at physiologic pressure in BD-ligated control animals (open circles) and in opossums after $1 \mathrm{~h}$ (closed circles) or $3 \mathrm{~h}$ (open triangles) of combined P-BD obstruction. Morphometric evaluation of the endocytic, secretory, and lysosomal compartments was executed as described in the text. In control animals, the vast majority of vesicles labeled with dextran 40 are found along the secretory pathway and only negligible amounts can be detected in structures of lysosomal origin. After P-BD obstruction, the secretory compartment remains practically unlabeled by dextran 40 reaction product. Lysosomal structures, on the other hand, progressively accumulate this content tracer. Asterisks indicate values significantly different ( $\alpha$ $\leq 0.05$ ) from the respective control values.

compared with the cat-ferritin group. The uptake and transport kinetics of this tracer were comparable to those of cat-ferritin.

Quantitation of uptake and targeting of dextran 40. Dextran 40 was found in endocytic vesicles with a frequency that was similar to that noted for cat-ferritin (Fig. 8). The subsequent targeting of this tracer, however, was found to be entirely different. Condensing vacuoles and secretory vesicles were the principal organelles found to contain the marker in control animals. At the earliest time interval studied ( $3 \mathrm{~min}$ ), significant amounts of dextran-positive secretory vesicles could be detected in the post-Golgi region of acinar cells. Lysosomes from control animals were only rarely observed to contain the tracer. This distribution was changed dramatically after P-BD ligation. Regardless of whether the ligature was performed 1 or $3 \mathrm{~h}$ before the instillation of tracer into the duct, organelles of lysosomal origin were progressively found to contain dextran reaction product, and the tracer disappeared almost completely from the secretory pathway.

Quantitation of uptake and targeting of $\left[{ }^{14} \mathrm{C}\right]$ dextran. The distribution of silver grains was similar to that previously reported for condensed dextran 40 . Whereas only a small number of grains were detected over endocytic vesicles, many grains were found over the secretory compartment of control animals 30 and $60 \mathrm{~min}$ after instillation. After $3 \mathrm{~h}$ of P-BD obstruction, the ratio of silver grains over secretory and lysosomal vesicles was reversed (Fig. 9). The lysosomal compartment became the principal target for $\left[{ }^{14} \mathrm{C}\right]$ dextran after luminal endocytosis.

\section{Discussion}

The pancreatic acinar cell is a well-characterized exocrine cell that secretes in a polarized and regulated manner. Its secretory

\section{ENDOCYTIC VESICLES}
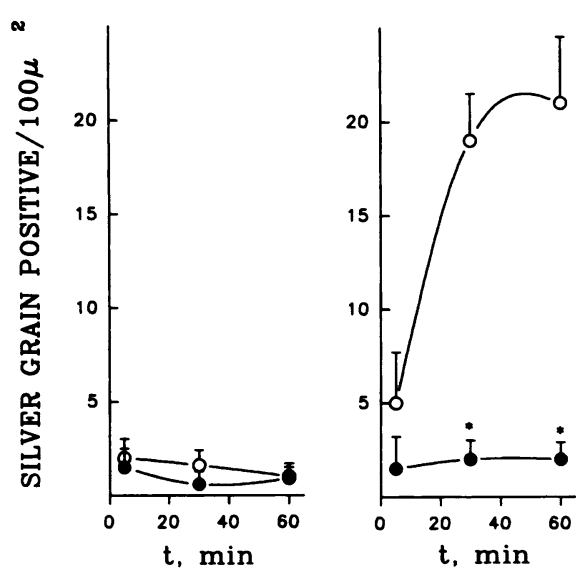

SECRETORY VESICLES

\section{LYSOSOMAL} VESICLES

Figure 9. Uptake and targeting of $\left[{ }^{14} \mathrm{C}\right]$ dextran in acinar cells. $\left[{ }^{14} \mathrm{C}\right]-$ dextran solution was instilled into the pancreatic duct at physiologic pressure in BD-ligated control animals (open circles) and in opossums after $3 \mathrm{~h}$ (closed circles) of combined P-BD obstruction. Morphometric evaluation of electron microscope autoradiographs was done as described in the text. In control animals the vast majority of silver grains were found over secretory vesicles, whereas after P-BD obstruction, lysosomes became the primary structure containing the radioactive label. Asterisks indicate values significantly different $(\alpha \leq 0.05)$ from the respective control values.

product, which consists of digestive enzyme zymogens, is stored in membrane-confined granules that are transported along microtubules to the apical pole of the cell, where their content is extruded into the lumen of the acinus after fusion/fission of the zymogen granule limiting membrane with the apical plasmalemma $(22,23)$. As a result, the granule membrane is incorporated into the plasmalemma. If mechanisms for removal of surface membrane were not operative, the cell membrane would rapidly increase in surface area. Retrieval of surface membranes, after exocytosis, is a mechanism through which this imbalance of membrane distribution is prevented (24). Surface membrane is rapidly recycled, via coated pits and vesicles, to Golgi cisternae (25) and other intracellular sites where it can be degraded and potentially reutilized. A distinct proportion of secreted digestive enzymes also appear to be reinternalized from the acinar lumen during the process of membrane retrieval (7).

Acute pancreatitis, a disease characterized by digestive necrosis of the gland, has been shown to be associated with premature, intraglandular activation of digestive proteases $(6,26,27)$. In view of the fact that acinar cells may reinternalize part of their secreted material, two issues must be considered. The first is the composition of the ductal content that might be internalized, and the second is the intracellular fate of that internalized material. In addition to digestive zymogens, acinar cells also secrete lysosomal hydrolases via both constitutive and regulated pathways (28). Lysosomal enzymes such as cathepsin B can activate digestive zymogens, even at a neutral pH (29), and during pancreatitis, activated digestive enzymes have been detected within the ductal space $(8-10)$. Thus, activated as well as potentially activatable zymogens may be present within the acinar lumen.

If in fact activated proteases, as opposed to inactive zymo- 

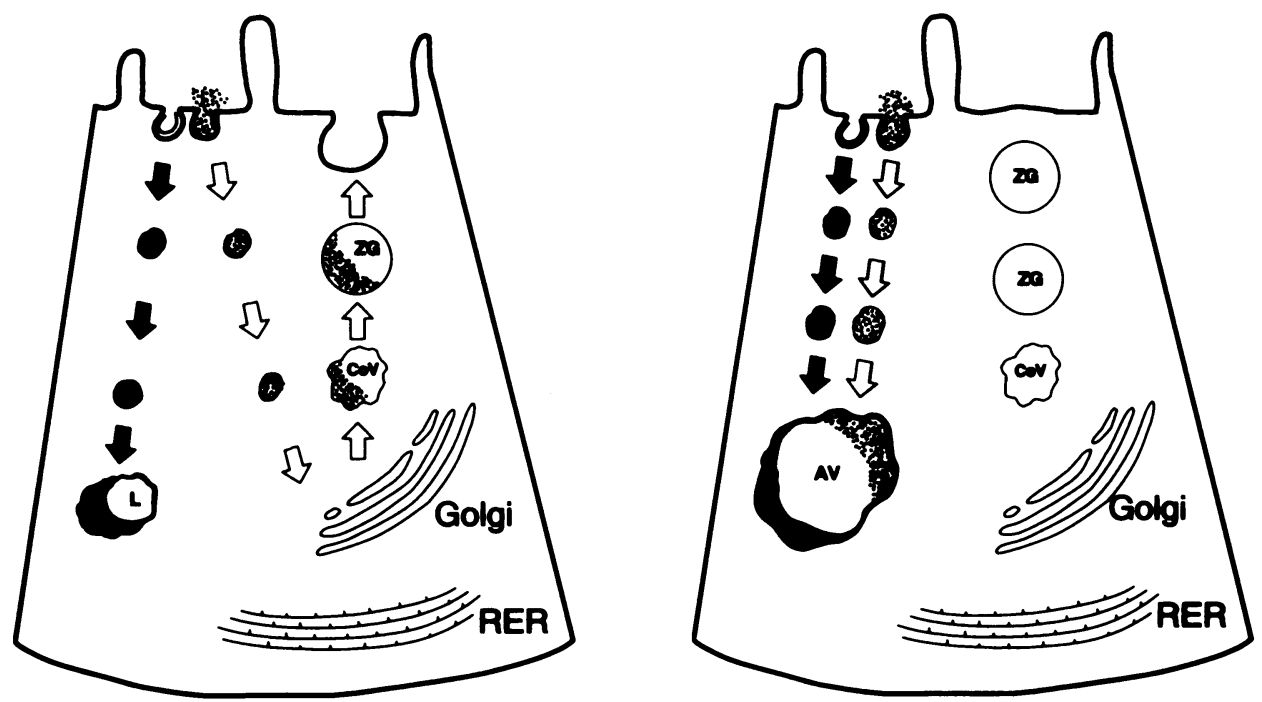

Figure 10. Pathways after endocytosis. The left panel indicates the physiologic situation. Resting acinar cells internalize tracer from the luminal plasmalemma. Markers for membrane-mediated endocytosis (cat-ferritin and HRP) are targeted to lysosomes $(L)$. Alternatively, the fluidphase tracer dextran is transported to the secretory pathway and rapidly reaches condensing vacuoles $(\mathrm{CoV})$ and zymogen granules $(Z G)$. The right panel indicates the situation after short-term P-BD ligation, a condition that eventually results in acute pancreatitis in the opossum. Endocytosis of tracer solution continues in the event of the mechanically blocked secretion. Irrespective of their binding characteristics at the luminal membrane, all three tracers are transported to the lysosomal compartment and are found in either lysosomes or autophagic vacuoles $(A V)$. Transport to the secretory compartment ceases after surgical duct ligation. $R E R$, rough endoplasmic reticulum.

gens, were internalized by acinar cells, the consequences might be deleterious. If, on the other hand, the inactive zymogens of proteases were wrongly targeted to an intracellular organelle where they could potentially be activated, such as a lysosome, instead of being reutilized in the secretory pathway (7), the result might be equally harmful.

We have studied the luminal uptake of three classes of marker molecules from the apical plasmalemma of pancreatic acinar cells. Tracers that bind to membrane domains by either affinity-mediated (cat-ferritin) or receptor-mediated (HRP) mechanisms undergo endocytosis and are subsequently transported to the lysosomal compartment. This observation is in accordance with previous studies on endocytosis in exocrine cells $(13,16)$. Prior P-BD obstruction, a condition that induces acute pancreatitis in the opossum $(3,11)$, does not arrest endocytosis, and the intracellular targeting and localization of these two tracers remain essentially unchanged. Dextran, on the other hand, is a marker that undergoes endocytosis along with bulk amounts of fluids without prior binding to membrane domains. Most of this tracer is subsequently transported into the secretory compartment of pancreatic acinar cells under physiologic conditions and accordingly behaves like a stable analogue of secretory enzymes after luminal endocytosis in the pancreas $(7,16)$. We have not investigated whether and how dextran could again be secreted from the cells, but the presence of the tracer in a regulated secretory compartment makes the assumption likely that it can, once again, exit the cell via exocytosis at the apex. After P-BD obstruction, and hence during the early stages of pancreatitis that precede acinar cell necrosis, the uptake of dextran from the lumen continues. The intracellular transport and targeting of this tracer, however, are dramatically altered. Only negligible amounts can be detected at the original site along the regulated secretory pathway, and the majority is translocated to the lysosomal compartment. Because the uptake and intracellular transport of the content marker dextran represent the uptake and transport of the physiologic content proteases, these altered intracellular kinetics could lead to a colocalization of lysosomal hydrolases with digestive proteases in a nonsecretable intracel- lular pool (Fig. 10). Alternatively, a blockage of the secretory pathway after P-BD obstruction could allow for bulk fluid endocytosis via a default pathway from the lumen. The observation that cellular polarity and apical morphology are altered early after duct obstruction (11) would offer a possible basis for a default pathway. However, regardless of whether only intracellular targeting or also the mechanism of endocytosis is altered, the consequence is an intracellular colocalization of lysosomal and digestive hydrolases. Such a colocalization has previously been observed in two unrelated models of pancreatitis, and we proposed it to be a critical event for the intracellular activation of proteases in pancreatitis $(30,31)$. In those studies, however, fusion of lysosomes with zymogen granules was thought to be the source of colocalization, whereas in the present investigation, uptake from the lumen appears as a novel alternative.

We have previously shown that the intracellular transport and sorting of proteins are markedly altered in early acute experimental pancreatitis (31) and after P-BD obstruction (32). During transport of newly synthesized enzymes through the cell, a missorting from the lysosomal into the regulated secretory pathway was observed (28). Interestingly, transport from the apex of the cell in the opposite direction also appears to be disturbed, but in this case, material that is targeted physiologically to the secretory pathway is now misdirected to the lysosomal compartment.

The experimental observations reported here were made within the first few hours after P-BD obstruction. Although there is now little doubt that pancreatic outflow obstruction rather than regurgitation of bile represents the triggering event for gallstone-induced pancreatitis also in humans $(5,33)$, questions regarding the degree and duration of obstruction required for the disease to develop remain unresolved. The majority of patients with pancreatitis are admitted to hospital only after the offending gallstone has already passed (34), and early relief of the obstruction has been shown to halt the progression of the disease $(35,36)$. However, an outflow obstruction of several hours, as in our experimental setting, is almost certain to have occurred in a patient with gallstone-induced pancreatitis. The 
amount of secretory protease that needs to be taken up and redirected inside an acinar cell before it reaches a critical level and exerts a harmful or deleterious effect remains equally unknown. In the opossum up to $3 \mathrm{ml}$ of secretory fluid can accumulate in the ductal space before secretion ceases, and this volume would be available for endocytosis, which we have shown to continue.

We conclude that endocytosis of membrane and content tracers from the apical lumen of pancreatic acinar cells continues after mechanical blockage of secretion. A content tracer that is physiologically translocated to the secretory compartment is targeted to lysosomes after P-BD obstruction. This mechanism represents a potential source for the cellular uptake of activated proteases and for the colocalization of digestive and lysosomal hydrolases during the early stages of acute pancreatitis that precede acinar cell necrosis. These events may play an important role in the initiation of cell injury during later phases of the disease.

\section{Acknowledgments}

The authors wish to thank E. Morgan for expert assistance with electron microscopic autoradiography and Ms. R. A. Monahan-Early and Dr. A. M. Dvorak for technical support and encouragement. We would also like to thank Ms. S. Gwin for superb secretarial assistance.

This work was supported by grants from the National Institutes of Health (31396) and from Deutsche Forschungsgemeinschaft (Le 625/ 1-2 and $\mathrm{Ru} 439 / 2-1$ ).

\section{References}

1. Acosta, J. L., and C. L. Ledesma. 1974. Gallstone migration as a cause of acute pancreatitis. $N$. Engl. J. Med. 290:484-487.

2. Opie, E. L. 1901. The etiology of acute hemorrhagic pancreatitis. Johns Hopkins Hosp. Bull. 12:182-188.

3. Lerch, M. M., A. Saluja, M. Rünzi, R. Dawra, M. Saluja, and M. L. Steer. 1993. Pancreatic duct obstruction triggers acute necrotizing pancreatitis in the opossum. Gastroenterology. 104:853-861.

4. Hernandez, C. A., and M. M. Lerch. 1993. Sphincter stenosis results from gallstone migration through the biliary tract. Lancet. 341:1371-1373.

5. Lerch, M. M., H. Weidenbach, C. A. Hernandez, G. Preclik, and G. Adler. 1994. Pancreatic outflow obstruction as the critical event for human gallstoneinduced pancreatitis. Gut. 35:1501-1503.

6. Leach, S. D., I. M. Modlin, G. A. Scheele, and F. S. Gorelick. 1991. Intracellular activation of digestive zymogens in rat pancreatic acini. Stimulation by high doses of cholecystokinin. J. Clin. Invest. 87:326-366.

7. Romagnoli, P., and V. Herzog. 1987. Reinternalization of secretory proteins during membrane recycling in rat pancreatic acinar cells. Eur. J. Cell Biol. 44:167-175.

8. Allan, B. J., R. Tournut, and T. T. White. 1973. Intraductal activation of human pancreatic zymogens. N. Engl. J. Med. 288:266.

9. Geokas, M. C., and H. Rinderknecht. 1974. Free proteolytic enzymes in pancreatic juice of patients with acute pancreatitis. Am. J. Dig. Dis. 19:591-598

10. Rinderknecht, H. 1986. Activation of pancreatic zymogens. Dig. Dis. Sci. 31:314-321.

11. Lerch, M. M., A. K. Saluja, R. Dawra, P. Ramarao, M. Saluja, and M. L. Steer. 1992. Acute necrotizing pancreatitis in the opossum: earliest morphologic changes involve acinar cells. Gastroenterology. 103:205-213.

12. Simonescu, N., M. Simonescu, and G. E. Palade. 1972. Permeability of intestinal capillaries. J. Cell Biol. 53:365-392.
13. Livine, E., and C. Oliver. 1986. Internalization of cationized ferritin by isolated pancreatic acinar cells. J. Histochem. Cytochem. 34:167-176.

14. Sung, S. S. J., R. S. Nelson, and S. C. Silverstein. 1983. The role of the mannose/ $\mathrm{N}$-acetylglucosamine receptor in the pinocytosis of horseradish peroxidase by mouse peritoneal macrophages. J. Cell. Physiol. 116:21-25.

15. Lang, T., and C. De Chastellier. 1985. Fluid phase and mannose receptormediated uptake of horseradish peroxidase in mouse bone marrow-derived macrophages. Biochemical and ultrastructural study. Biol. Cell. 53:149-159.

16. Herzog, V., and H. Reggio. 1980. Pathways of endocytosis from luminal membrane in rat exocrine pancreas. Eur. J. Cell Biol. 21:141-150.

17. Graham, R. C., and M. J. Karnovsky. 1966. The early stages of absorption of injected horseradish peroxidase in the proximal tubules of mouse kidney. Ultrastructural cytochemistry by a new technique. J. Histochem. Cytochem. 14:291-302.

18. Novikoff, A. B., M. Mori, N. Quintana, and A. Yam. 1978. Study on the secretory process in the mammalian exocrine pancreas. I. The condensing vacuoles. J. Cell Biol. 75:148-165.

19. Salpeter, M. M., and M. L. Bachmann. 1964. Autoradiography with the electron microscope. A procedure for improving resolution, sensitivity, and contrast. J. Cell Biol. 49:877-882.

20. Weibel, E. R. 1973. Stereological techniques for electron microscopic morphometry. In Principles and Techniques of Electron Microscopy. M. A. Hyat, editor. Von Nostrad Reinhold, New York. 237-296.

21. Nadler, N. J. 1971. The interpretation of grain counts in electron microscope radioautography. J. Cell Biol. 49:877-882.

22. Jamieson, J. D., and G. E. Palade. 1971. Synthesis, intracellular transport, and discharge of secretory proteins in stimulated pancreatic exocrine cells. J. Cell Biol. 50:135-158.

23. Beaudoin, A. R., and G. Grondin. 1991. Secretory pathways in animal cells: with emphasis on pancreatic acinar cells. J. Electron Microsc. Tech. 71:5169.

24. Koike, H., and J. Meldolesi. 1981. Post-stimulation retrieval of luminal surface membrane in parotid acinar cells is calcium-dependent. Exp. Cell Res. 134:377-388.

25. Herzog, V., and M. G. Farquhar. 1977. Luminal membrane retrieved after exocytosis reaches most Golgi cisternae in secretory cells. Proc. Natl. Acad. Sci. USA. 74:5073-5077.

26. Yamaguchi, H., T. Kimura, K. Mimura, and H. Nawata. 1989. Activation of proteases in caerulein-induced pancreatitis. Pancreas. 4:565-571.

27. Bialek, R., S. Willemer, R. Arnold, and G. Adler. 1991. Evidence of intracellular activation of serine proteases in acute caerulein-induced pancreatitis in rats. Scand. J. Gastroenterol. 26:190-196.

28. Hirano, T., A. Saluja, P. Ramarao, M. M. Lerch, M. Saluja, and M. L. Steer. 1991. Apical secretion of lysosomal enzymes in rabbit pancreas occurs via a secretagogue regulated pathway and is increased after pancreatic duct obstruction. J. Clin. Invest. 87:864-869.

29. Lerch, M. M., A. K. Saluja, R. Dawra, M. Saluja, and M. L. Steer. 1993. The effect of chloroquine administration in two different models of acute pancreatitis. Gastroenterology. 104:1768-1779.

30. Saluja, A., S. Hashimoto, M. Saluja, R. E. Powers, J. Meldolesi, and M. L. Steer. 1987. Subcellular redistribution of lysosomal enzymes during caerulein-induced pancreatitis. Am. J. Physiol. 246:G457-G467.

31. Saluja, M., A. Saluja, M. M. Lerch, and M. L. Steer. 1991. A plasma protease which is expressed during supramaximal stimulation causes in vitro subcellular redistribution of lysosomal enzymes in rat exocrine pancreas. J. Clin. Invest. 87:1280-1285.

32. Saluja, A., M. Saluja, A. Villa, U. Leli, P. Rutledge, J. Meldolesi, and M. L. Steer. 1989. Pancreatic duct obstruction in rabbits causes digestive zymogen and lysosomal enzyme colocalization. J. Clin. Invest. 84:1260-1266.

33. Lerch, M. M., C. A. Hernandez, and G. Adler. 1994. Acute pancreatitis. N. Engl. J. Med. 331:948-949.

34. Acosta, J. M., C. A. Pellegrini, and D. B. Skinner. 1980. Etiology and pathogenesis of acute biliary pancreatitis. Surgery. 88:118-125.

35. Rünzi, M., A. Saluja, M. M. Lerch, R. Dawra, H. Nishino, and M. L. Steer. 1993. Early ductal decompression prevents the progression of biliary pancreatitis: an experimental study in the opossum. Gastroenterology. 105:157-164.

36. Neoptolemos, J. P., D. L. Carr-Locke, N. J. London, I. A. Bailey, D. James, and D. P. Fossard. 1988. Controlled trial of urgent endoscopic retrograde cholangio-pancreatography versus conservative treatment for acute pancreatitis due to gallstones. Lancet. 2:979-983. 